\title{
Study on the Method of Fatigue Test Loading Spectrum of the Connecting Rod in Diesel Engine
}

\author{
Qing-guo LUO ${ }^{1, a}$, Xu-dong WANG ${ }^{1, b}$, Geng-yun ZHANG ${ }^{1, c}$, Feng WANG ${ }^{2, d}$ \\ and Shu-sheng BAI ${ }^{3, \mathrm{e}}$
}

${ }^{1}$ Department of Mechanical Engineering, Academy of Armored Force Engineering, Beijing, 100072, China

${ }^{2}$ Beijing Martial Delegate Agency, Headquarters of the General Equipment, Beijing, 100042, China

${ }^{3}$ In Liyang Machinery Co., Ltd. Military Representative Office, Anshun, 561102, China

aluogingguozgy@yahoo.com.cn, ${ }^{b}$ wangxudong3637@163.com, 'zgy718@sina.com, 'shangmachangcun@126.com, ${ }^{\mathrm{e}}$ baishu sheng@163.com

Keywords: Connecting rod, Rigid-flexible coupling, Loading spectrum, Fatigue test

\begin{abstract}
Based on flexible multibody system dynamic theory, founded the rigid-flexible coupling multibody dynamic analysis model of the crank and connecting rod mechanism of diesel engine which included the piston, link, crankshaft and flywheel. The dynamic stress of the connecting rod was calculated and counted by the rain flow method. Counted the stress histories of the connecting rod at all the mission profiles, and then extrapolate and superpose the rain flow matrix, get the stress cumulative frequency distribution of the dangerous node. The load spectrum for the fatigue test of the connecting rod was established, and the mew method can be used for making the specification of the fatigue test of the connecting rod. The load spectrum for connecting rod fatigue test, which can reflect the fatigue life more accurate.
\end{abstract}

\section{Introduction}

Crank and connecting rod mechanism is the most important moving parts of diesel engine, which changes the reciprocating motion of the piston to rotation of the crankshaft and export torque. In the diesel motive cycle, crank and connecting rod mechanism stress periodic cylinder pressure, reciprocating inertia force, rotary centrifugal force and torque, which bending and torsion the crankshaft. With modern diesel power, density continues to increase the working conditions of crank and connecting rod mechanism increasingly harsh, so that checking the strength of moving parts and test the fatigue life, have become the key step in the engine reliability design and verification.

In the periodic dynamic loading, the fatigue rupture of the connecting rod is high frequency fatigue. In the conventional method of fatigue test, the test loads are defined by the max tension and compressive stress multiply with a factor according to the standard of the industry and enterprise, so the stress amplitude is invariant. But the operation condition of engine is invariant only in the bench test, and it always variable in actually of using because of the road ramp angle and the driver's operation. The stress of the connecting rod is related with the engine speed and the max cylinder pressure that are variable in the working conditions. So the fatigue test with invariable load amplitude can't reflect the fatigue life of the connecting rod.

In this paper, crank and connecting rod mechanism is the research object; a rigid-flexible coupling dynamic simulation model is established based on rigid-flexible multi-body modeling theory. The dynamic stress of the connecting rod was calculated and counted by the rain flow method, and the load spectrum for the fatigue test of the connecting rod was established.

\section{Basic theory of flexible body dynamic}

Flexible body is plasmodium, the relative position of each point inside changes all the time, so leading the flexible coordinate system to describe the relative deformation of each point inside the 
flexible body. Then the motion of each point in the flexible body can be described as a resultant movement of the rigid motion of the standard coordinate system and the elastic deformation. For any point $\mathrm{P}$ of the flexible body, whose position vector is:

$$
r=r_{0}+A\left(s_{p}+u_{p}\right)
$$

Where, $r$ is the vector of point $\mathrm{P}$ in the inertial coordinate system, $r_{0}$ is the vector of the initial point in the inertial coordinate system, $A$ is the matrix of direction cosine, $s_{p}$ is the vector of point $\mathrm{P}$ in the floating coordinate system undeformed, $u_{p}$ is the vector of relative deformation.

Flexible body equations of motion based on Lagrangian differential equations is this form as follows:

$$
M \xi+N_{\xi} \frac{1}{2}\left[\frac{\partial M}{\partial \xi} \xi^{T} \xi \psi_{+} K \xi+f_{g}+D \xi \psi_{4}\left[\frac{\partial \Psi}{\partial \xi}\right]^{T} \lambda=Q\right.
$$

Where, $\xi, \xi \&$ is the generalized coordinates for the flexible body and its time derivative, $M, N$ is the mass matrix of flexible body and its time derivative, $\frac{\partial M}{\partial \xi}$ is the mass matrix of flexible body generalized coordinates of the partial derivatives.

\section{Rigid-flexible coupling model}

Crankshaft and connecting rod are complicated elastic continuum, and its flexible feature plays an important role for the dynamics character of the diesel engine. In order to consider the elastic deformation of the connecting rod and improve the precision of dynamics simulation, we should establish the rigid-flexible coupling model. The model contains the flexible crankshaft and connecting rod; piston assembly, flywheel rigid model; constraint relationship between the various components and the loads effect on the system components.

Using the three-dimensional modeling software to establish the full-scale model of the connecting rod, then output the model to the finite element analysis software. Defining the material properties, meshing and doing other pre-treatment work, and the mesh model of the connecting rod is shown in Fig.1.

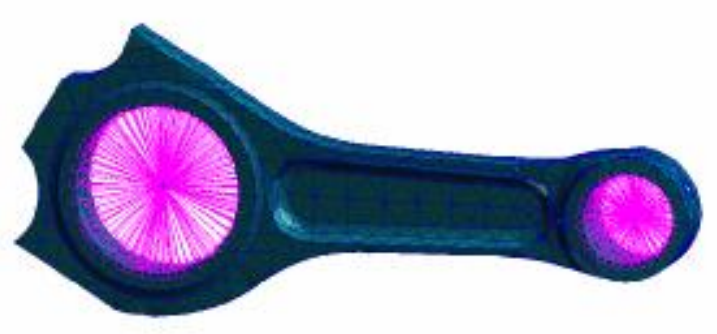

Fig.1 Mesh model of connecting rod

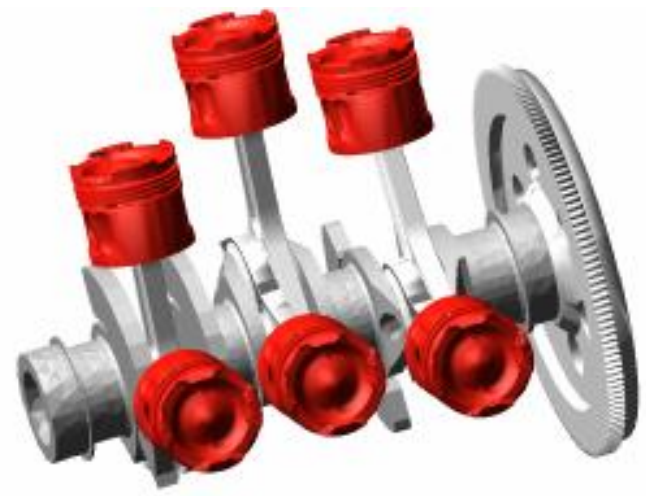

Fig.2 Rigid-flexible coupling model

Then analysis the free modal of crankshaft and output of the modal neutral file (*. mnf), which contains the material, cell, node, stress and strain information of the crankshaft.

The rigid body dynamics models include the crankshaft, connecting rod group, the piston group and the flywheel. After the modal analysis, neutral file can be generated, and then replacing the original rigid crankshaft and connecting rods. The rigid-flexible coupling model of crank and connecting rod mechanism is shown in Fig 2.

According to the actual movements of the mechanism, the author sets the motion pairs to the common constraints. For example, the revolute joint were used in such cases: the main bearing of the crankshaft, the joint between connecting rod and crankshaft, the joint between connecting rod and piston pin; the translational joint was used between the piston and cylinder; fixed joint was used between crankshaft and flywheel. 
The gas pressure act on the top of piston of each cylinder should be applied according to the order of explosion stroke such as left 1 , right 1 , left 2 , right 2 , left 3 , and right 3 by the crank angle as the independent variable.

\section{Dynamic simulation and result}

In a working cycle, the stress of the connecting rod is alternating tension and compression. The max compression stress is at the time of the outbreak of the cylinder, the stress nephogram is shown as Fig 3, the stress concentrators are near the arc transitions of the crankpin end and the piston pin end. In order to research the relationship between the stress of the connecting rod and operation condition of the engine, the following stress operation conditions are selected to be simulated, they are the minimum power condition, the maximum torque condition and the maximum power condition.

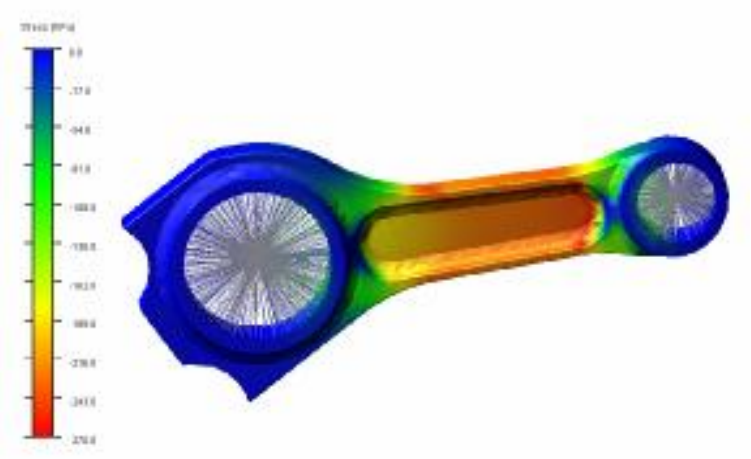

Fig.3 Stress nephogram of the connecting rod

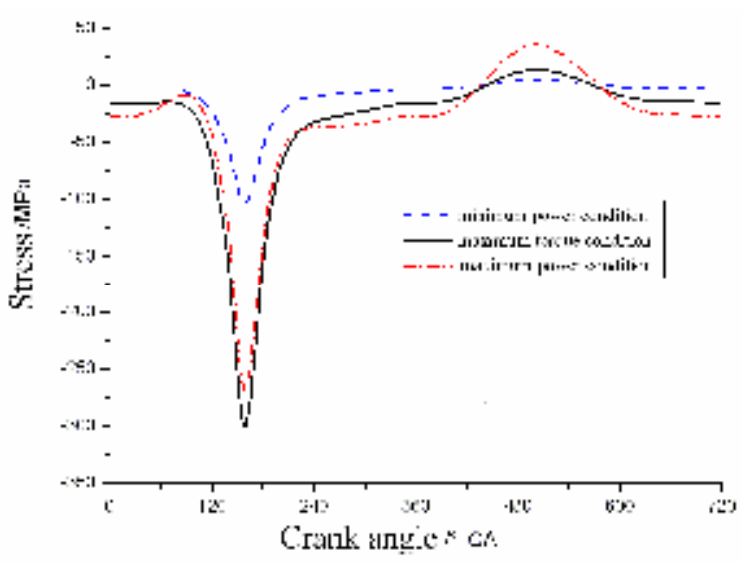

Fig.4 Stress of the dangerous node

The experimental cylinder pressure data were used as the boundary condition, steady-state simulated for a work cycle, the number of simulation steps is 720 , and the stress of the dangerous node of the connecting rod is shown as Fig 4.

When the cylinder is working at compression travel and power stroke, the stress of the connecting rod is compressive stress, which is negative in the Fig 4; and when the cylinder is working at the TDC of the input stroke and exhaust stroke, the cylinder pressure is less than the inertia force of the piston, so the stress of the connecting rod is tensile stress, which is positive in the Fig 4.

Table 1 The stress of the dangerous node in different operation condition

\begin{tabular}{c|c|c|c|c}
\hline Operation condition & $\begin{array}{c}\text { Enginespeed } \\
(\mathrm{r} / \mathrm{min})\end{array}$ & $\begin{array}{c}\text { Max tensile stress } \\
(\mathrm{Mpa})\end{array}$ & $\begin{array}{c}\text { Max compressive stress } \\
\mathrm{MPa}\end{array}$ & $\begin{array}{c}\text { Stress amplitude } \\
\mathrm{MPa}\end{array}$ \\
\hline minimum power condition & 800 & 5.51 & -100.6 & 106.11 \\
\hline maximum torque condition & 1500 & 14.4 & -300 & 314.4 \\
\hline maximum power condition & 2200 & 35.8 & -268 & 303.8
\end{tabular}

The stress of the dangerous node in different operation condition is shown in Table 1. When the engine is working at the minimum power condition, the cylinder pressure and the engine speed are both minimum and the stress amplitude is only $106.11 \mathrm{MPa}$. When the engine is working at the maximum torque condition, the cylinder pressure is maximum and the stress amplitude of the dangerous node is $314.4 \mathrm{MPa}$. When the engine is working at the maximum power condition, the cylinder pressure is less than the maximum torque condition but the max tensile stress is $35.8 \mathrm{MPa}$ because of a high engine speed, and the stress amplitude of the dangerous node is $303.8 \mathrm{MPa}$. When the engine is working between the maximum power condition and the maximum torque condition, the stress amplitude will exceed the amplitude of invariable operation condition, and the maximum amplitude can be exceed to $335.8 \mathrm{MPa}$.

This shows that the mechanical load of the connecting rod is related to the cylinder pressure and inertia force, and the amplitude is variable with the operation condition of the engine. According to the specification and the test data of the vehicle type approval, we can get the operation condition histories of the engine. 


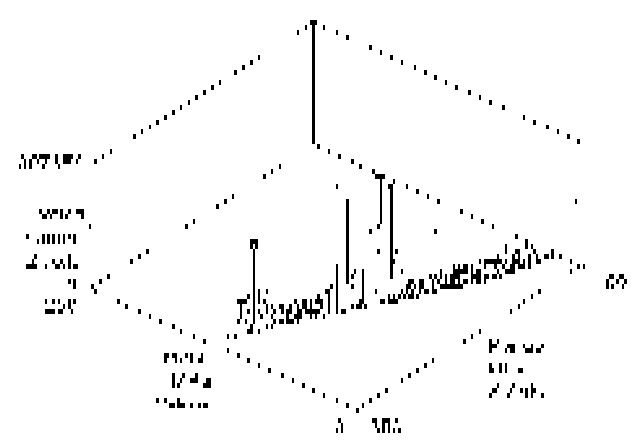

Fig.5 Counting matrix of rain flow

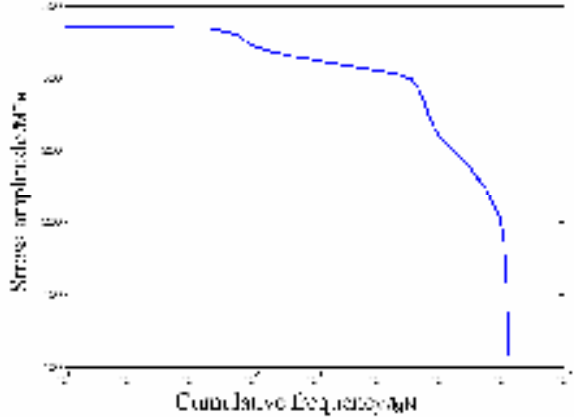

Fig.6 Stress cumulative frequency distribution

Take the wavy road in warm zone for example; the operation condition of the engine is variable during the driving. The cylinder pressure and engine speed are loaded as the boundary condition, then the stress history of the dangerous node of the connecting rod can be calculated based on the rigid-flexible multi-body modeling. Then counting the stress history by rain flow method and the rain flow matrix of the connecting rod on the wavy road in warm zone is shown in Fig5.

Counting the stress histories of the connecting rod at other mission profiles, and then extrapolate and superpose the rain flow matrix, the stress cumulative frequency distribution of the dangerous node is shown as Fig 6, and it also can be defined as the load spectrum.

Table 2 The load spectrum for connecting rod fatigue test

\begin{tabular}{c|c|c|c|c|c|c|c|c}
\hline Load grade & 1 & 2 & 3 & 4 & 5 & 6 & 7 & 8 \\
\hline Stress amplitude/ $\mathrm{MPa}$ & 336 & 324 & 300 & 271 & 236 & 200 & 165 & 129 \\
\hline Load frequency & 239 & 721 & 325360 & 443480 & 2797300 & 6437900 & 2175000 & 1854000 \\
\hline Cumulative frequency & 239 & 960 & 326320 & 769800 & 3567100 & 10005000 & 12180000 & 14034000 \\
\hline
\end{tabular}

The stress can be divided into 8 grades to fitting the stress cumulative frequency distribution. The scale coefficients are $1,0.95,0.85,0.725,0.575,0.425,0.275$ and 0.125 . The load spectrum for connecting rod fatigue test is shown as Table 2 , which can reflect the fatigue life more accurately.

\section{Conclusions}

(1) The dynamic stress of the connecting rod was calculated based on the finite element and multibody dynamic theory.

(2) Counted the stress histories of the connecting rod at all the mission profiles, and then extrapolate and superpose the rain flow matrix, get the stress cumulative frequency distribution of the dangerous node.

(3) Established the load spectrum for connecting rod fatigue test, which can reflect the fatigue life more accurate.

\section{References}

[1] Wubo. Numerical simulation study on fatigue test of the connecting rod in diesel Engine[J].Small internal combustion engine and motorcycle. 2010, 39(5):47-50

[2] Lili Ting, Wei Li: Diesel engine, 2007, (3):5-8

[3] Xingguo Ma, Xiaomei You: Journal of vibration and Shock, 2008, (9):155-157

[4] Aibing Zhu, Lei Guo: Journal of machine design, Vol.25 (2008):31-33 